\title{
Web Review: Picture archiving and communication system (PACS)
}

\author{
IK Indrajit \\ Department of Radiodiagnosis and Imaging Command Hospital (Air Force), Bangalore, Karnataka, India
}

Correspondence: Dr. IK Indrajit, Department of Radiodiagnosis and Imaging Command Hospital (Air Force), Bangalore - 560007, Karnataka, India. E-mail: inji63@gmail.com

A few useful websites dealing with PACS in radiology that are available at the moment on the Internet are reviewed below.

1. PACS History at http://www.pacshistory.org/index. html offers documents 'that describe the history of the development of picture archiving and communications systems (PACS), teleradiology, medical image displays, and related technology, such as compression and medical image communication standards like DICOM.' Available sections include Documents, Pictures, and Videos.

2. The Society for Imaging Informatics in Medicine (SIIM) at http://www.siimweb.org/ is an important resource for imaging professionals interested in the current and future use of technologies, including PACS. Previously called Society for Computer Applications in Radiology (SCAR) until 2006, it has many interesting sections. A few key references to the history of digital imaging in medicine are available at http://www. siimweb.org/index.cfm?id=7538. A compendium of 'top $10^{\prime}$ lists is available at http://www.siimweb.org/index. $\mathrm{cfm}$ ? $\mathrm{id}=2558$. A typical illustrative example is 'Top 10 Reasons Why PACS Go Down' available at http://www. siimweb.org/index.cfm?id=1144.

3. NHS Connecting for Health (NHS CFH) has its PACS page at http://www.connectingforhealth.nhs.uk/ systemsandservices/pacs/. Available in this webpage are sections such as Learn more about PACS, Latest PACS news, PACS beyond 2013, PACS and voice recognition, and Frequently asked questions.

4. OTech at http://www.otechimg.com/index.cfm is a healthcare technology portal providing PACS training

\begin{tabular}{|l|l|}
\hline \multicolumn{2}{|c|}{ Access this article online } \\
\hline Quick Response Code: & \\
\hline & Website: \\
\hline & www.iji.org \\
\hline
\end{tabular}

and consulting for PACS administrators and other professionals. Educative books, study guides, computerbased training, seminars, newsletters, and hands-on workshops on a range of topics from DICOM and HL7 to PACS system administration and advanced PACS troubleshooting techniques are available. The webportal has sections such as Training, Publications, Professional Services, and Products. Recent educative items include PACS Policy Manual, Ver. 3, and Troubleshooting/ Monitoring Hardware and Software Tools.

5. Image Management and PACS at http://users.erols. com/veader/ is a portal for 'exchanging information among radiology professionals regarding PACS implementation.' Sections that are on offer include Benefits, Justification, Implementing, Archives, Communications Missing Pieces, Configurations, and Integration Issues.

6. Radiology PACS Administrator Site at http://www. pacsadminforum.com/ provides information for the PACS administrator. Issues regarding implementing PACS, finding PACS gear, impact of PACS on workflow, PACS teleradiology, PACS administration training, etc., are covered. Many sections such as PACS News, PACS Tools, Nighthawk Services, Education, Resources and Articles are available.

7. A PACS Administrators and Imaging Informatics Professional Resource Guide is a handy document which is available from the Atlanta Rad Tech website at http://atlantaradtech.org/upload/1263271008_PACS\%20 Admin\%20and\%20IIP\%20Resource\%20Guide\%20 2008fnl.pdf.

8. PACS Primer is a copyrighted educative material authored by P. Berman, Lumisys, Inc and is available from http://rad.usuhs.mil/project44R3/download/ pacs_primer.pdf. The material, though written in the late nineties, offers a basic insight into PACS and its building blocks, e.g., the workstation, networking, and input/output devices. Things you need to know when embarking on a PACS project is available at http://www. brit.com/Product $\% 20$ Sheet $\% 20$ PDFs/BRIT\%20PACS $\% 20$ 101.pdf 
9. PACS White Papers are available from the multinational vendor Intelerad at http:/ / fr.intelerad.com/en/white_ papers.php as a part of the 'Learning' section. The topics covered currently include PACS Primer: Hardware and Networking Jargon, PACS and IT: The Reality, PACS Terminology: A primer's way around the jargon, Under the Hood: RIS/PACS integrations, Steps to a successful PACS implementation, and The 'Ins and Outs' of data migration. Similarly white papers from Health Imaging Hub is available at http:/ / www.healthimaginghub. com/downloads/white-papers.html .

10. PACSnet Storage Calculator at http://www. ninestepstougly.com/pacsnet.org.uk/calc2.html is a useful online tool that can calculate the storage requirements of modalities at radiology departments. The modalities include plain film, fluoroscopy, USG, CT scan, MRI, angiography, nuclear medicine, and mammography. By entering modalities and procedures from a list, the daily number of images and appropriate size in MB are shown. A'calculate' button calculates the results and offers the total file sizes in MB for a day, week, month, or year. A note of caution, however the values derived from the table are for guidance only and offer only typical values; to obtain actual values, one may need to contact the vendors.

\section{Endpiece}

Few blogs and webpages from PACS experts are available as in Herman Oosterwijk's corner at http:// www.healthimaginghub.com/hermans-corner.html, Dalai"s PACS Blogs at http://doctordalai.blogspot.com/ Medical Imaging Blog RIS/PACS sources at http:// www.medicalimagingtalk.com and PACS World Blog for radiology network administrators at http://pacsworld. blogspot.com/search/label/PACS.

CT History at http://www.impactscan.org/CThistory.htm offers a nutshell view of the progress that has occurred in CT technology over the years. Sections on scientific background and early technical development, clinical acceptance and early commercial development, are available, as a timeline from 1895-2011. A link to an interesting article 'The Story Behind the Development of the First Whole-body Computerized Tomography Scanner' by Robert S. Ledley et al. from J Am Med Inform Assoc is linked here and available also at http://www.ncbi.nlm.nih.gov/pmc/articles/ PMC1561796/pdf/465.0600124. 